\title{
La estructura de capital en las medianas empresas del departamento de Boyacá, Colombia
}

Capital structure in medium-sized companies in Department of Boyacá, Colombia

\section{Estrutura de capital das médias empresas em departamento de Boyacá, Colombia}

\footnotetext{
Magíster en Administración, Contador Público. Profesor Asociado del Departamento de Contaduría Pública en la Universidad de Bogotá Jorge Tadeo Lozano, Bogotá, Colombia. Dirección de correspondencia: Carrera 4 No. 22-61, Módulo 1 Oficina 529, Bogotá, Colombia. Correo electrónico:andresf.mejiaa@utadeo.edu.co
} 


\section{Resumen}

El estudio de la estructura de capital es uno de los tópicos financieros de mayor impacto, puesto que entre las teorías que intentan explicarla todavía no se ha llegado a un consenso sobre las determinantes y el comportamiento de las decisiones de financiamiento en las empresas. La investigación sobre el comportamiento de la estructura de capital en las empresas de tamaño medio y pequeño es un asunto aún más complejo, pues los modelos tradicionales son aplicables principalmente a empresas que se cotizan en los mercados de capitales o son de interés público.

Para este trabajo se seleccionaron las medianas empresas en el departamento de Boyacá en Colombia como población por estudiar, aplicando un modelo que se ha utilizado en casos similares en otros países; se demostró empíricamente la preferencia por la financiación con recursos propios antes que con deuda, la cual, a su vez, se prefirió por las empresas con mayor volatilidad en su rentabilidad y por las de mayor crecimiento.

Palabras clave: estructura de capital, medianas empresas, financiamiento.

Clasificación JEL: C33, G32, M21

\section{Abstract}

The study of capital structure is one of the financial topics of greatest impact, since among the theories that attempt to explain it,has not yet reached a consensus on the determinants and the behavior of funding decisions in companies. Research on the behavior of capital structure in small and medium size companies, is even more complex issue, since traditional models are mainly applicable to trade on the capital markets or in public interest companies.

For this work midsize companies were selected, in the Department of Boyacá in Colombia as study population, applying a model that has been used in similar cases in other countries; in the study has empirically demonstrated preference for equity financing rather than debt, which in turn was preferred by companies with greater volatility in profitability and fastest growing.

Keywords: capital structure, medium sized companies, funding.

\section{Resumo}

O estudo da estrutura de capital é um dos assuntos financeiros de maior impacto, uma vez que entre as teorias que tentam explicar ainda não chegaram a um consenso sobre os determinantes e o comportamento das decisões de financiamento 
das empresas. A pesquisa sobre o comportamento da estrutura de capital em tamanho médio e pequeno porte das empresas é uma questão mais complexa, uma vez que os modelos tradicionais são principalmente aplicáveis ao comércio nos mercados de capitais ou nas empresas de interesse público.

Para esse trabalho, empresas de médio porte foram selecionadas no Departamento de Boyacá, na Colômbia como população de estudo, a aplicação de um modelo que tem sido usada em casos semelhantes em outros países; empiricamente demonstrado preferência para financiamento de capital em vez de com a dívida, que por sua vez foi preferido por empresas com maior volatilidade da rentabilidade e de crescimento mais rápido.

Palavras-chave: Estrutura de capital, financiamento de médias empresas.

\section{INTRODUCCIÓN}

El financiamiento de las empresas ha suscitado diversos tópicos de estudio, teniendo en cuenta que es la representación de los recursos que sustentan su existencia y operación; además, porque de su origen, distribución, manejo y gestión depende en gran medida su desempeño general. Adicionalmente, la selección y mezcla adecuada de fuentes de financiamiento han sido asociadas, entre otros conceptos, a la generación de valor como cumplimiento pleno del objetivo de la función financiera en las organizaciones.

En Colombia hay alrededor de un $99 \%$ de micro, pequeñas y medianas empresas, frente a solamente un $1 \%$ de grandes empresas, de ahí que cumplan un importante papel como dinamizadoras de la economía, generadoras de empleo y demandantes de recursos del mercado financiero para el logro de su éxito empresarial (Comisión Económica para América Latina y el Caribe CEPAL, 2011). La situación en el departamento de Boyacá es similar en cuanto a la distribución. De acuerdo con los datos del SIREM, se encuentra alrededor de un $98 \%$ de micro, pequeñas y medianas frente a un $2 \%$ de grandes empresas.

El acceso a los recursos financieros por parte de las pequeñas y medianas empresas es un asunto complejo, puesto que existen barreras que las propias dinámicas del mercado se encargan de crear, y que no son fácilmente superables, especialmente en los primeros años de vida de las empresas, o por la calidad de la información contable y financiera que envían como señal de su 
solidez al mercado, o por el alto riesgo que implica financiarlas, lo que encarece los recursos y aleja las posibilidades de recibir financiación externa.

De otra parte, los empresarios en este tipo de organizaciones, frecuentemente son adversos al endeudamiento, porque desconocen la forma adecuada de beneficiarse de esta fuente de financiación y consideran que los recursos propios son la mejor opción, lo que limita su crecimiento a la posibilidad de generar tales recursos, con el sacrificio de posibilidades de crecimiento y de otras ventajas que puede brindar el endeudamiento adecuadamente utilizado.

Se observa que para el año 2010, en las pymes colombianas, la principal fuente de financiación para las inversiones fueron los recursos propios, con alrededor del $68 \%$ de contribución, seguida de lejos por el endeudamiento bancario, con aproximadamente el $13 \%$; mientras que para el promedio general del país, los recursos propios se situaron en el $44 \%$ y los préstamos bancarios en un $21 \%$ (The World Bank \& Internacional Finance Corporation, 2011).

En este trabajo se han tomado como referentes las medianas empresas del departamento de Boyacá, teniendo en cuenta que dentro del grupo de pymes son las empresas con información contable y financiera de mejor calidad, se encuentran en una etapa de mayor madurez y tienen un impacto económico más representativo en la economía regional que las micro y pequeñas. Se utilizó un panel de datos para cinco años (2007-2011), se aplicaron modelos para medir la determinación en el endeudamiento total, el de largo plazo y la estructura financiera o proporción deuda/recursos propios.

Para el caso de las pequeñas y medianas empresas, no es factible utilizar los modelos tradicionales que utilizan variables del mercado de capitales, por lo que algunos autores han desarrollado y aplicado modelos con variables alternativas, que se basan en la información contable y en razones construidas con ella.

El propósito de realizar el estudio es conocer la forma en que se han financiado las empresas, caracterizar su estructura de capital y observar las principales determinantes de sus decisiones de financiamiento de acuerdo con variables y modelos que han sido utilizados en otros países (desarrollados y en desarrollo), específicamente para pymes, para validar sus conclusiones o descartarlas, a través de su prueba empírica.

\section{MARCO DE REFERENCIA}

\section{Marco teórico}

Con el fin de contextualizar adecuadamente este trabajo, es indispensable partir de la evolución de los conceptos sobre la estructura de capital, las teorías que han intentado explicar su comportamiento y los desarrollos empíricos que se han dado al respecto; por lo tanto se parte del trabajo seminal de Miller y Modigliani, que data de la década de los cincuenta 
del siglo pasado, conocido como tesis de la irrelevancia de Miller y Modigliani (1958), teoría que inicia la discusión contemporánea en dicha temática.

El planteamiento fundamental de Miller y Modigliani en su primera teoría, tiene que ver con la irrelevancia de la estructura de capital de las empresas en su valor, es decir, que independientemente de la distribución de las fuentes de financiamiento, el valor no cambia en una situación ideal de mercados perfectos, planteamiento que iba en contra de las tesis de las finanzas tradicionales de ese entonces.

Para la década siguiente, los mismos autores formulan una segunda teoría (Miller \& Modigliani, 1963) que constituye una corrección a su primera versión, y en la que consideran que, en presencia de impuestos, circunstancia ajena al mundo ideal de mercados perfectos, las empresas pueden aprovechar lo que denominaron "escudo fiscal de la deuda" o ventajas obtenidas por la deducibilidad de los intereses para efectos de impuestos, lo que implicaría el uso de recursos obtenidos como deuda financiera, circunstancia en la cual la estructura de capital no sería irrelevante.

Posteriormente se desarrollan dos teorías principales, que explican de manera diferente la decisión sobre la estructura de capital, la teoría del trade-off y la del pecking-order.

La teoría del trade-off (Bradley, Jarrell \& Kim, 1984; Frank \& Goyal, 2008), o del óptimo financiero, concluye que la empresa se ajusta en el tiempo a una estructura óptima de capital que crea un equilibrio perfecto entre los costes de quiebra y el escudo fiscal de la deuda, del que ya habían hablado Miller y Modigliani (1963). De acuerdo con esta teoría, el ajuste se da en un lapso de tiempo diferente para cada empresa.

La teoría del pecking-order (Myers,1984; Myers \& Majluf, 1984) se contrapone a la anterior. Los autores criticaron su capacidad explicativa, puesto que, según ellos, las empresas tienen una jerarquía a la hora de escoger fuentes de financiamiento, partiendo de los recursos propios y teniendo como última posibilidad la emisión de acciones, basándose en la asimetría de información (Shyam-Sunder \& Myers, 1998).

Por otra parte, la teoría del comportamiento de sincronización con el mercado o market-timing behavior, explica la estructura de capital en función de las señales que da el mercado a las compañías, por lo que las empresas tienden a realizar emisiones de acciones cuando se percibe un comportamiento favorable en el mercado (Baker \& Wurgler, 2002) y presentan tendencia a recomprar sus propias acciones cuando los valores de mercado están más bajos, situaciones que evidencian una relación fuerte entre la estructura de capital y los comportamientos históricos del mercado de capitales.

Se observa, entonces, como la disyuntiva en la investigación sobre la estructura 
de capital se ha centrado en las teorías de la jerarquía de preferencias (pecking order) y el óptimo financiero (trade-off), en modelos que buscan demostrar empíricamente las dos teorías, determinantes y comportamientos de las mismas que se han asociado a cada teoría, aunque más recientemente algunos autores han planteado que antes que ser teorías opuestas, son complementarias y que por sí mismas, ni individual, ni conjuntamente, tienen una capacidad explicativa completa del fenómeno de la estructura de capital.

En este sentido se replantea la teoría del óptimo financiero, que por naturaleza es estático, a una teoría del óptimo financiero dinámico, que se consigue a lo largo de los años y que para cada empresa tendrá una velocidad de ajuste propia, de acuerdo con las observaciones empíricas de varios autores (Dang, Kim \& Shin, 2012), (Qian, Tian \& Wirjanto, 2009), (Morellec, Nikolov \& Schürnoff, 2012), (Boot \& Thakor, 2011). De otra parte, otros autores han hablado de un efecto no observable, que genera estructuras de capital estables en el tiempo y que no ha sido explicado por los modelos $\mathrm{y}$ determinantes tradicionales utilizados hasta ahora, valga decir, las teorías de la irrelevancia, con su segunda proposición donde incluye el efecto fiscal de la deuda, la teoría de la jerarquía de preferencias (pecking-order), la del óptimo financiero (trade-off), y la de las señales del mercado, entre otras (Lemmon, Roberts \& Zender, 2008; Leary, 2009).

\section{La estructura de capital en las pyme}

El estudio de la estructura de capital, su comportamiento y determinantes, ha sido abordado tradicionalmente para grandes empresas, o para aquellas cuyos títulos se cotizan en los mercados de capitales o que son de interés público, entre otras razones porque se dispone de mayor información sobre su comportamiento y variables; esto obedece a que en estas condiciones son más observables ciertas tendencias, que permiten inferir conclusiones con respecto a sus decisiones de financiación.

Sin embargo, en Colombia, como en muchos otros países, las pymes son el tipo de empresa predominante y para estas es limitado el acceso a fuentes de financiamiento, tales como la emisión de deuda (bonos) o acciones, por el precario desarrollo de los mercados de capitales, así como tampoco están extendidos los fondos de capital de riesgo o los inversionistas "ángeles", que patrocinan pequeñas iniciativas de empresa con el objeto de obtener rentabilidades futuras, que no se han desarrollado en Colombia por las condiciones institucionales, que son distintas a las de países como por ejemplo Estados Unidos (Barona \& Gómez, 2010, p. 88), donde los ángeles inversionistas son una alternativa extendida de impulso al emprendimiento e ideas de empresa.

El acceso al crédito ha sido una de las limitaciones más importantes para las pymes en el país (Stephanou \& 
Rodríguez, 2008). Con base en una encuesta de Fundes de 2003 practicada en 687 pymes, el acceso al financiamiento se clasificó como la segunda restricción más importante (particularmente para empresas más pequeñas, industriales y más jóvenes) en la creación, desarrollo o diversificación de sus actividades económicas. Dentro de la categoría del acceso al financiamiento, las condiciones de crédito, relacionadas principalmente con tasa de interés, plazo, requerimientos de garantía y procedimientos para otorgar préstamos, se percibieron como los factores limitantes más significativos (Stephanou \& Rodríguez, 2008).

En cuanto al estudio empírico de la estructura de capital en las pymes, se encuentran referentes, como el caso de las pymes de propiedad familiar en México (Berumen, García \& Domenge, 2012), donde se utilizó un modelo con determinantes de la estructura de capital o variables independientes, tales como tamaño de la empresa, planeación administrativa y estratégica formal, control familiar, edad del director general, edad o antigüedad de la empresa, y las variables de análisis de la estructura de capital,que son las dependientes, en donde se tuvieron en cuenta la deuda, los préstamos familiares, el capital social y las utilidades acumuladas (financiación con recursos propios); igualmente, se utilizaron hipótesis que interrelacionan las variables del modelo.

Allí se concluyó que especialmente las características de los propietarios y gerentes, como experiencia, expectativas fi- nancieras, aversión al riesgo, actitud ante el control y variables externas macro y microeconómicas, legales, tecnológicas, etc., tienen influencia en las decisiones de financiamiento.

En el estudio de Forte, Barros y Nakamura (2013), se analizó la estructura de capital en las pequeñas y medianas empresas brasileras, utilizando variables como rentabilidad, estructura y crecimiento de los activos, edad, riesgo, crecimiento, escudos fiscales diferentes a la deuda, y se encontraron relaciones de carácter negativo con la rentabilidad, positivo con el crecimiento de los activos, negativo con la edad y negativo con el riesgo.

Para el caso de las pymes en España, se utilizó un modelo econométrico (AybarArias, Casino-Martínez \& López-Gracia, 2012) con base en una hipótesis de ajuste dinámico a una estructura de capital óptima (dynamic trade-off). Como determinantes se emplearon el riesgo de quiebra, las oportunidades de crecimiento, la rentabilidad, los escudos fiscales diferentes a la deuda, la tangibilidad de los activos, la singularidad de sus productos y el tamaño; como variable dependiente se usó el valor de mercado o valor en libros de la deuda.

Como resultado de este estudio se encontró que las determinantes de la velocidad de ajuste, se relacionan con la distancia entre la deuda óptima y la observada, las tasas de flexibilidad financiera, las oportunidades de crecimiento y el tamaño, demostrándose un ajuste parcial 
del modelo, debido principalmente a las variables características de las empresas que han probado tradicionalmente su relación con la estructura de capital (Aybar-Arias et al., 2012).

En el estudio de Psillaki y Daskalakis (2009), se evaluó si las determinantes de la estructura de capital tienen origen únicamente en la firma o existen algunas del país o entorno institucional. Para este fin se realizó un comparativo entre pequeñas y medianas empresas de Grecia, Francia, Portugal e Italia, en el que se utilizaron variables como la estructura de activos, tamaño, rentabilidad, riesgo y oportunidades de crecimiento; se concluyó que las determinantes que tuvieron relaciones estadísticamente significativas con las decisiones de endeudamiento, se originaron en las empresas, no en las condiciones institucionales.

Otro referente es el estudio de PalacínSánchez, Ramírez Herrera y Di Pietro (2013), sobre la estructura de capital en las pymes en distintas regiones españolas, en el que se buscó identificar posibles factores regionales dentro del mismo país como determinantes de las decisiones de financiación; se concluyó que las variables endógenas de las empresas son las que presentan relaciones estadísticamente significativas, descartando diferencias asociadas a las condiciones regionales en el mismo país. Las variables que probaron empíricamente su relación fueron tamaño, estructura de activos, rentabilidad, crecimiento y edad.
Actividad empresarial en el departamento de Boyacá

El departamento de Boyacá se encuentra en la región andina, en el centro-oriente del país, tiene una geografía variada en cuanto a pisos térmicos y allí se desarrollan diferentes actividades económicas, centradas principalmente en el sector agropecuario (cultivos de caña panelera y papa, especialmente), minero, servicios sociales, comunales y personales y manufactura (Ministerio de Comercio, Industria y Turismo, 2012).

De acuerdo con el Ministerio de Comercio, Industria y Turismo (2012), en el perfil económico del departamento de Boyacá se encuentra que aportó para el año 2012 un 2,8\% del producto interno bruto nacional, su PIB per cápita se situó en 8.216 dólares, por encima del promedio nacional (Ministerio de Comercio, Industria y Turismo, 2014).

En cuanto a la complejidad de trámites para la apertura de una empresa, la ciudad de Tunja ocupó el puesto 22 entre 23 capitales de departamento estudiadas en nivel de complejidad; es decir, una de las ciudades que requirió mayores trámites a la hora de establecer nuevos negocios (Ministerio de Comercio, Industria y Turismo, 2014).

Las condiciones de desempleo del departamento se reflejan en la tasa de desempleo en la ciudad de Tunja, su capital, que de manera sostenida desde 2007 se ha mantenido por encima de la media de las 
capitales de departamento del país, ubicándose en un $11,9 \%$ hacia el año 2012 (Departamento Administrativo Nacional de Estadística DANE, 2012; Ministerio de Comercio, Industria y Turismo, 2014).

En cuanto a las actividades de comercio exterior, el departamento participa en un muy reducido porcentaje en las exportaciones nacionales $(0,7 \%$ del total en 2012), que provienen principalmente del sector industrial, con un $95,9 \%$ del total del departamento para 2012 (DANE, 2012).

Siendo Boyacá una región de Colombia, que es un país de micro, pequeñas y medianas empresas (mipymes), como puede deducirse a partir de la base de datos SIREM de la Superintendencia de Sociedades, la más grande del país, donde el $84 \%$ de empresas informantes son mi pymes, y dentro de este grupo la proporción de pequeñas y medianas es prácticamente igual, con un $49 \%$ de pequeñas y $48 \%$ de medianas empresas, algunas micro (un $3 \%$ ) también reportaron, la situación del departamento es similar. Sin embargo hay un gran volumen de empresas que no reportan a esta base de datos, principalmente micro y pequeñas, puesto que su inspección se escapa a la acción de dicha entidad. Los criterios de tamaño en este artículo corresponden a los incluidos en la Ley 905 de 2004.

\section{Modelos estadísticos}

Para el presente estudio se ha seleccionado como método de análisis estadístico la regresión lineal múltiple, en tres diferentes modelos con tres diferentes variables dependientes. La primera mide el endeudamiento total (pasivos) frente a los activos totales (Aybar Arias et al., 2012; Psillaki \& Daskalakis, 2009; Forte et al. 2013); la segunda, el endeudamiento de largo plazo (pasivos de largo plazo o no corrientes) frente a los activos totales (Forte et al. 2013); y la tercera, que se ha denominado estructura financiera, mide la proporción de deuda frente a recursos propios (pasivos / patrimonio):

1. Endeudamiento total

$$
\text { Endeudamiento total }=\frac{\text { Pasivo corriente }+ \text { Pasivo no corriente }}{\text { Activo total }}
$$

2. Endeudamiento de largo plazo

$$
\text { Endeudamiento de largo plazo }=\frac{\text { Pasivo no corriente }}{\text { Activo total }}
$$

3. Estructura financiera

$$
\text { Estructura financiera }=\frac{\text { Pasivo total }}{\text { Patrimonio }}
$$


De la misma forma se han definido como variables independientes o determinantes de la estructura, el riesgo, medido como la desviación estándar o volatilidad de la rentabilidad durante los periodos analizados (Aybar-Arias et al., 2012; Forte et al. , 2013); el crecimiento de la empresa, medido en términos del crecimiento comparativo de las ventas cada año frente al periodo inmediatamente anterior (Macas-Nunes, Serrasqueiro, Nunes \& Mendes, 2013; Aybar-Arias et al., 2012; Forte et al., 2013); una segunda razón del crecimiento, la razón de variación de los activos totales de cada periodo, frente al inmediatamente anterior; la rentabilidad, que es la razón entre las ventas y los activos totales; una segunda medida de la rentabilidad, el rendimiento sobre el patrimonio (ROE), obtenido a partir de la razón entre la utilidad neta y el patrimonio (Aybar-Arias et al., 2012; Forte et al., 2013); el tamaño como logaritmo natural de los activos totales (Forte et al., 2013); la estructura de activos, que se obtiene a partir de la razón entre los gastos por depreciación del período y los activos totales (Forte et al.,2013); los escudos fiscales distintos a deuda (Aybar-Arias et al., 2012; Forte et al., 2013), que son la razón entre los gastos por depreciación del período y las ventas totales; y la tangibilidad de los activos, que es la razón entre los activos fijos (no corrientes) y los activos totales (Macas-Nunes et al.,2013; Aybar-Arias et al., 2012; Psillaki \& Daskalakis, 2009). Estos indicadores se detallan a continuación, así:

\section{Riesgo}

$$
\text { Riesgo }=\sigma \text { Rentabilidad }
$$

2. Crecimiento en ventas

$$
\text { Crecimiento en ventas }=\frac{\text { Ventas }_{t}-\text { Ventas }_{t-1}}{\text { Ventas }_{t-1}}
$$

3. Crecimiento de los activos

$$
\text { Crecimiento de los activos }=\frac{{\text { Activos } \text { totales }_{t}-\text { Activos }_{\text {totales }}}_{t-1}}{{\text { Activos } \text { totales }_{t-1}}}
$$

4. Rentabilidad

$$
\text { Rentabilidad }=\frac{\text { Utilidad neta }}{\text { Patrimonio }}
$$


5. Rendimiento sobre el patrimonio

$$
\text { Rendimiento sobre el patrimonio }=\frac{\text { Utilidad neta }}{\text { Patrimonio }}
$$

6. Tamaño

$$
\text { Tamaño }=\text { Ln Activos }
$$

7. Estructura de activos

$$
\text { Estructura de activos }=\frac{\text { Gastos en depreciación }}{\text { Activos totales }}
$$

8. Escudos fiscales diferentes a la deuda

Escudos fiscales diferentes a la deuda $=\frac{\text { Depreciación }}{\text { Ventas }}$

La información del panel utilizado corresponde a una muestra de 23 empresas medianas, de la población total que corresponde para 2007 a 29 empresas, cuyos estados financieros se encuentran en la base de datos del SIREM de la Superintendencia de Sociedades, de las cuales se descartaron 6 por no presentar información completa durante el periodo analizado.

Las empresas están domiciliadas en el departamento de Boyacá para el periodo 2007-2011, y cumplen con los criterios de la Ley 905 de 2004, particularmente en lo relacionado con el volumen de activos totales, que para este caso se encuentra en el rango entre 5.001 y 30.000 salarios mínimos mensuales legales vigentes. Este parámetro se tuvo en cuenta para la selección de la muestra en el periodo inicial (2007) y la muestra es uniforme para los cinco años.

Los estadísticos descriptivos de las variables involucradas en el modelo se encuentran en la Tabla 1.

En el primer modelo, la variable dependiente es el endeudamiento total, se incluyen todas las variables independientes, sus resultados se muestran en la Tabla 2. Se encontró una bondad de ajuste de 0,819 , de acuerdo con el coeficiente $R^{2}$, que indica su capacidad explicativa. El resultado hallado en este coeficiente indica mayor bondad de ajuste en tanto se aproxime más a 1 . 
Tabla 1. Estadísticos descriptivos de las variables.

\begin{tabular}{|l|c|c|c|}
\hline \multicolumn{4}{c|}{ Estadísticos descriptivos } \\
\hline Media & Desviación estándar & $\mathbf{N}^{\circ}$ & \\
\hline Endeudamiento total & 0,502653529710079 & 0,227813653725725 & 23 \\
Crecimiento en ventas & 0,350600648383734 & 0,989000610821130 & 23 \\
Crecimiento de los activos & 0,190904955250379 & 0,159234979426692 & 23 \\
Rentabilidad & 2,364971995697793 & 2,121487001264890 & 23 \\
Rendimiento sobre el patrimonio & 0,122091362626387 & 0,130054170494874 & 23 \\
Tamaño & 15,611956277145028 & 0,530670940688165 & 23 \\
Estructura de activos & 0,020548431469226 & 0,020673190384137 & 23 \\
Escudos fiscales diferentes a deuda & 0,012010088283481 & 0,012335349510545 & 23 \\
Riesgo & 0,386484701680191 & 0,248599882577948 & 23 \\
Tangiblidad de los activos & 0,3857 & 0,21175 & 23 \\
\hline
\end{tabular}

Fuente: SPSS, elaboración del autor

Tabla 2. Resumen del modelo 1.

\begin{tabular}{|c|c|c|c|c|c|c|c|c|c|c|}
\hline \multicolumn{11}{|c|}{ Resumen del modelo $^{b}$} \\
\hline \multirow[b]{2}{*}{ Modelo } & \multirow[b]{2}{*}{$\mathrm{R}$} & \multirow[b]{2}{*}{$\begin{array}{c}\mathrm{R} \\
\text { Cuadrado }\end{array}$} & \multirow[b]{2}{*}{$\begin{array}{c}\mathrm{R} \\
\text { Cuadrado } \\
\text { Ajustado }\end{array}$} & \multirow[b]{2}{*}{$\begin{array}{c}\text { Error estándar } \\
\text { de la } \\
\text { estimación }\end{array}$} & \multicolumn{5}{|c|}{ Estadísticas de cambios } & \multirow[b]{2}{*}{$\begin{array}{l}\text { Durbin- } \\
\text { Watson }\end{array}$} \\
\hline & & & & & $\begin{array}{c}\text { Cambio de } \\
\text { Cuadrado } \\
\text { de R } \\
0,819\end{array}$ & $\begin{array}{c}\text { Cambio } \\
\text { en F }\end{array}$ & df1 & df2 & $\begin{array}{l}\text { Sig. } \\
\text { Cambio } \\
\text { en F }\end{array}$ & \\
\hline 1 & $0,905^{\mathrm{a}}$ & 0,819 & 0,693 & ,126153199458343 & & 6,527 & 9 & 13 & 0,001 & 1,885 \\
\hline \multicolumn{11}{|c|}{$\begin{array}{l}\text { a. Predictores: (Constante), Tang. Activos, Prom Sales Growth, Risk, Prom ROE, Prom Depr Exp, Prom LN Asset, } \\
\text { Prom Asset Growth, Prom Non Debt Tax Shields, Prom Profitability }\end{array}$} \\
\hline
\end{tabular}

Fuente: SPSS, elaboración del autor.

Tabla 3. Análisis de la varianza del modelo 1.

\begin{tabular}{|c|l|c|c|c|c|c|}
\hline \multicolumn{7}{|c|}{ ANOVA $^{\text {a }}$} \\
\hline \multicolumn{2}{|c|}{ Modelo } & Suma de cuadrados & gl & Media cuadrática & F & Sig. \\
\hline \multicolumn{1}{|c|}{1} & Regresión & 0,935 & 9 & 0,104 & 6,527 & $0,001 \mathrm{~b}$ \\
& Residuo & 0,207 & 13 & 0,016 & & \\
& Total & 1,142 & 22 & & & \\
\hline
\end{tabular}

a. Variable dependiente: PromTotal leverage

b. Predictores: (Constante), Tang. Activos, Prom Sales Growth, Risk, Prom ROE, Prom Depr Exp, Prom LN Asset, Prom Asset Growth, Prom Non Debt Tax Shields, Prom Profitability

Fuente: SPSS, elaboración del autor 
Apuntes Cenes Vol. 33, Nº. 58, ISSN 0120-3053

enero - junio 2015, Págs. 185-206

Tabla 4. Coeficientes del modelo 1.

\begin{tabular}{|c|c|c|c|c|c|c|}
\hline \multicolumn{7}{|c|}{ Coeficientes $^{\mathrm{a}}$} \\
\hline & \multirow{2}{*}{ Modelo } & \multicolumn{2}{|c|}{$\begin{array}{l}\text { Coeficientes no } \\
\text { estandarizados }\end{array}$} & \multirow{2}{*}{\begin{tabular}{|c|}
$\begin{array}{c}\text { Coeficientes estan- } \\
\text { darizados }\end{array}$ \\
Beta
\end{tabular}} & \multirow[b]{2}{*}{$\mathrm{t}$} & \multirow[b]{2}{*}{ Sig. } \\
\hline & & B & $\begin{array}{l}\text { Error } \\
\text { estándar }\end{array}$ & & & \\
\hline \multirow[t]{10}{*}{1} & (Constante) & $-0,564$ & 1,052 & & $-0,536$ & 0,601 \\
\hline & PromSalesGrowth & 0,054 & 0,033 & 0,233 & 1,646 & 0,124 \\
\hline & PromAssetGrowth & $-0,245$ & 0,227 & $-0,171$ & $-1,075$ & 0,302 \\
\hline & PromProfitability & $-0,025$ & 0,052 & $-0,231$ & $-0,473$ & 0,644 \\
\hline & PromROE & 0,453 & 0,228 & 0,258 & 1,983 & 0,069 \\
\hline & PromLNAsset & 0,073 & 0,069 & 0,170 & 1,066 & 0,306 \\
\hline & PromDeprExp & 0,279 & 4,674 & 0,025 & 0,060 & 0,953 \\
\hline & PromNonDebtTaxShields & 3,414 & 5,042 & 0,185 & 0,677 & 0,510 \\
\hline & Risk & 0,563 & 0,191 & 0,614 & 2,953 & 0,011 \\
\hline & Tang.Activos & $-0,796$ & 0,150 & $-0,740$ & $-5,292$ & 0,000 \\
\hline
\end{tabular}

Fuente: SPSS, elaboración del autor

Según los resultados del modelo 1 , aplicado para el endeudamiento total, las variables que tuvieron una relación importante como determinantes fueron la tangibilidad de los activos y el riesgo, de acuerdo con la prueba de significancia estadística, como puede observarse en la Tabla 4. La relación del riesgo con el endeudamiento total es de carácter positivo, mientras que la tangibilidad tiene una relación de signo negativo.
Este resultado muestra que el incremento del riesgo en las empresas, y por lo tanto aquellas que presentaron un mayor nivel de riesgo, tuvieron un mayor nivel de endeudamiento total; dado que el riesgo se midió como la desviación estándar de la rentabilidad de los diferentes periodos, se puede afirmar que las empresas con mayor estabilidad en su rentabilidad, es decir, las que en consecuencia tuvieron menor riesgo, también presentaron menor endeudamiento, frente a aquellas donde se presentó mayor volatilidad. 
Tabla 5. Resumen del modelo 2.

\begin{tabular}{|c|c|c|c|c|c|c|c|c|c|c|}
\hline \multicolumn{11}{|c|}{ Resumen del modelo $2^{\text {b }}$} \\
\hline \multirow[b]{2}{*}{ Modelo } & \multirow[b]{2}{*}{$\mathrm{R}$} & \multirow[b]{2}{*}{$\begin{array}{c}\mathrm{R} \\
\text { Cuadrado }\end{array}$} & \multirow[b]{2}{*}{$\begin{array}{c}\mathrm{R} \\
\text { Cuadrado } \\
\text { Ajustado }\end{array}$} & \multirow[b]{2}{*}{$\begin{array}{c}\text { Error estándar } \\
\text { de la } \\
\text { estimación }\end{array}$} & \multicolumn{5}{|c|}{ Estadísticas de cambios } & \multirow[b]{2}{*}{$\begin{array}{l}\text { Durbin- } \\
\text { Watson }\end{array}$} \\
\hline & & & & & $\begin{array}{c}\text { Cambio de } \\
\text { Cuadrado } \\
\text { de R } \\
0,768\end{array}$ & $\begin{array}{c}\text { Cambio } \\
\text { en F }\end{array}$ & df1 & df2 & $\begin{array}{l}\text { Sig. } \\
\text { Cambio } \\
\text { en F }\end{array}$ & \\
\hline 1 & $0,905^{\mathrm{a}}$ & 0,768 & 0,608 & 0,065360717676557 & & 4,785 & 9 & 13 & ,006 & 2,022 \\
\hline \multicolumn{11}{|c|}{$\begin{array}{l}\text { a. Predictores: (Constante), Tang. Activos, Prom Sales Growth, Risk, Prom ROE, Prom Depr Exp, Prom LN Asset, } \\
\text { Prom Asset Growth, Prom Non Debt Tax Shields, Prom Profitability }\end{array}$} \\
\hline
\end{tabular}

Fuente: SPSS, elaboración del autor

El segundo modelo buscó explicar la correlación entre las variables y la determinación sobre la estructura de capital de largo plazo, con este fin se utilizó como variable independiente el endeudamiento de largo plazo, este modelo también presentó una considerable bondad de ajuste o capacidad explicativa, con un coeficiente de determinación de 0,768 .

Tabla 6. Análisis de varianza del modelo 2.

\begin{tabular}{|c|c|c|c|c|c|c|}
\hline \multicolumn{7}{|c|}{$\mathrm{ANOVA}^{\mathrm{a}}$} \\
\hline \multicolumn{2}{|c|}{ Modelo } & Suma de cuadrados & gl & Media cuadrática & $\mathrm{F}$ & Sig. \\
\hline 1 & Regresión & 0,184 & 9 & 0,020 & 4,785 & $0,006^{\mathrm{b}}$ \\
\hline & Residuo & 0,056 & 13 & 0,004 & & \\
\hline & Total & 0,240 & 22 & & & \\
\hline
\end{tabular}

a. Variable dependiente: Prom LT Leverage

b. Predictores: (Constante), Tang. Activos, Prom Sales Growth, Risk, Prom ROE, Prom Depr Exp, Prom LN Asset, Prom Asset Growth, Prom Non Debt Tax Shields, Prom Profitability

Fuente: SPSS, elaboración del autor 
Apuntes Cenes Vol. 33, №. 58, ISSN 0120-3053

enero - junio 2015, Págs. 185-206

Tabla 7. Coeficientes del modelo 2.

\begin{tabular}{|c|c|c|c|c|c|c|}
\hline \multicolumn{7}{|c|}{ Coeficientes $^{\mathrm{a}}$} \\
\hline & \multirow{2}{*}{ Modelo } & \multicolumn{2}{|c|}{$\begin{array}{l}\text { Coeficientes no } \\
\text { estandarizados }\end{array}$} & \multirow{2}{*}{\begin{tabular}{|c}
$\begin{array}{c}\text { Coeficientes estan- } \\
\text { darizados }\end{array}$ \\
Beta
\end{tabular}} & \multirow[b]{2}{*}{$\mathrm{t}$} & \multirow[b]{2}{*}{ Sig. } \\
\hline & & $\mathrm{B}$ & $\begin{array}{l}\text { Error } \\
\text { estándar }\end{array}$ & & & \\
\hline \multirow[t]{10}{*}{1} & (Constante) & $-0,281$ & 0,545 & & $-0,515$ & 0,615 \\
\hline & PromSalesGrowth & 0,083 & 0,017 & 0,785 & 4,895 & 0,000 \\
\hline & PromAssetGrowth & $-0,035$ & 0,118 & $-0,053$ & $-0,295$ & 0,772 \\
\hline & PromProfitability & $-0,014$ & 0,027 & $-0,287$ & $-0,520$ & 0,612 \\
\hline & PromROE & 0,133 & 0,118 & 0,166 & 1,124 & 0,281 \\
\hline & PromLNAsset & 0,017 & 0,036 & 0,087 & 0,481 & 0,639 \\
\hline & PromDeprExp & 0,291 & 2,422 & 0,058 & 0,120 & 0,906 \\
\hline & PromNonDebtTaxShield\$ & 1,498 & 2,613 & 0,177 & 0,573 & 0,576 \\
\hline & Risk & 0,118 & 0,099 & 0,282 & 1,198 & 0,252 \\
\hline & Tang.Activos & $-0,004$ & 0,078 & $-0,008$ & $-0,051$ & 0,960 \\
\hline
\end{tabular}

Fuente: SPSS, elaboración del autor

Para el caso del endeudamiento de largo plazo se observó una relación estadísticamente significativa con la variable crecimiento, medida a partir del crecimiento comparativo de las ventas de periodo a periodo, el cual presentó una relación marcada de carácter positivo, lo que indica que las empresas que tuvieron un mayor crecimiento en términos de ventas, a su vez presentaron un mayor endeudamiento de largo plazo. Se considera un hallazgo importante, puesto que indica que las empresas que utilizan las ventajas provenientes del endeudamiento, pueden alcanzar un mayor crecimiento, incrementar sus resultados y consolidarse, debido a las ventajas fiscales de la deuda.

Tabla 8. Resumen del modelo 3.

\begin{tabular}{|c|c|c|c|c|c|c|c|c|c|c|}
\hline \multicolumn{11}{|c|}{ Resumen del modelo $^{b}$} \\
\hline \multirow[b]{2}{*}{ Modelo } & \multirow[b]{2}{*}{$\mathrm{R}$} & \multirow[b]{2}{*}{$\begin{array}{c}\mathrm{R} \\
\text { Cuadrado }\end{array}$} & \multirow[b]{2}{*}{$\begin{array}{c}\text { R } \\
\text { Cuadrado } \\
\text { Ajustado }\end{array}$} & \multirow[b]{2}{*}{$\begin{array}{c}\text { Error estándar } \\
\text { de la } \\
\text { estimación }\end{array}$} & \multicolumn{5}{|c|}{ Estadísticas de cambios } & \multirow[b]{2}{*}{\begin{tabular}{|l} 
Durbin- \\
Watson
\end{tabular}} \\
\hline & & & & & $\begin{array}{c}\text { Cambio de } \\
\text { Cuadrado } \\
\text { de R } \\
0,803\end{array}$ & $\begin{array}{l}\text { Cambio } \\
\text { en F }\end{array}$ & df1 & df 2 & \begin{tabular}{|c|} 
Sig. \\
Cambio \\
en F
\end{tabular} & \\
\hline 1 & $0,896^{\mathrm{a}}$ & 0,803 & ,666 & 0,72953 & & 5,881 & 9 & 13 & 0,002 & 2,245 \\
\hline \multicolumn{11}{|c|}{$\begin{array}{l}\text { a. Predictores: (Constante), Tang.Activos, PromSalesGrowth, Risk, PromROE, PromDeprExp, PromLNAsset, } \\
\text { PromAssetGrowth, PromNonDebtTaxShields, PromProfitability }\end{array}$} \\
\hline b. Variabl & le depen & diente: Est & & & & & & & & \\
\hline
\end{tabular}

Fuente: SPSS, elaboración del autor 
En el tercer modelo se utilizó como variable dependiente la estructura financiera, razón entre los pasivos totales y el patrimonio total. Para este, el coeficiente de determinación mostró una considerable bondad de ajuste del modelo, como explicativo del comportamiento de la estructura de endeudamiento de las empresas. La razón seleccionada mide la proporción de recursos externos frente a los propios en el financiamiento de la operación de las empresas.

Tabla 9. Análisis de varianza del modelo 3.

\begin{tabular}{|c|c|c|c|c|c|c|}
\hline \multicolumn{7}{|c|}{ ANOVA $^{\mathrm{a}}$} \\
\hline \multicolumn{2}{|c|}{ Modelo } & Suma de cuadrados & gl & Media cuadrática & $\mathrm{F}$ & Sig. \\
\hline 1 & Regresión & 28,168 & 9 & 3,130 & 5,881 &, $002^{b}$ \\
\hline & Residuo & 6,919 & 13 &, 532 & & \\
\hline & Total & 35,086 & 22 & & & \\
\hline \multicolumn{7}{|c|}{ a. Variable dependiente: Estr_Fin } \\
\hline \multicolumn{7}{|c|}{$\begin{array}{l}\text { b. Predictores: (Constante), Tang.Activos, PromSalesGrowth, Risk, PromROE, Prom De- } \\
\text { prExp, PromLNAsset, PromAssetGrowth, PromNonDebtTaxShields, PromProfitability }\end{array}$} \\
\hline
\end{tabular}

Fuente: SPSS, elaboración del autor

Tabla 10. Coeficientes del modelo 3 .

\begin{tabular}{|c|c|c|c|c|c|c|}
\hline \multicolumn{7}{|c|}{ Coeficientes $^{\mathrm{a}}$} \\
\hline \multirow{2}{*}{\multicolumn{2}{|c|}{ Modelo }} & \multicolumn{2}{|c|}{$\begin{array}{l}\text { Coeficientes no } \\
\text { estandarizados }\end{array}$} & \multirow{2}{*}{\begin{tabular}{|c|}
$\begin{array}{c}\text { Coeficientes estan- } \\
\text { darizados }\end{array}$ \\
Beta
\end{tabular}} & \multirow[b]{2}{*}{$\mathrm{t}$} & \multirow[b]{2}{*}{ Sig. } \\
\hline & & B & $\begin{array}{c}\text { Error } \\
\text { estándar }\end{array}$ & & & \\
\hline \multirow[t]{10}{*}{1} & (Constante) & 2,260 & 6,082 & & 0,372 & 0,716 \\
\hline & PromSalesGrowth & 0,239 & 0,189 & 0,187 & 1,267 & 0,227 \\
\hline & PromAssetGrowth & $-1,223$ & 1,315 & $-0,154$ & $-0,930$ & 0,369 \\
\hline & PromProfitability & $-0,232$ & 0,303 & $-0,390$ & $-0,765$ & 0,458 \\
\hline & PromROE & 5,610 & 1,320 & 0,578 & 4,251 & 0,001 \\
\hline & PromLNAsset & $-0,059$ & 0,396 & $-0,025$ & $-0,148$ & 0,885 \\
\hline & PromDeprExp & $-2,733$ & 27,031 & $-0,045$ & $-0,101$ & 0,921 \\
\hline & PromNonDebtTaxShield $\$$ & 26,428 & 29,160 & 0,258 & 0,906 & 0,381 \\
\hline & Risk & 3,091 & 1,102 & 0,609 & 2,804 & 0,015 \\
\hline & Tang.Activos & $-3,130$ & 0,870 & $-0,525$ & $-3,598$ & 0,003 \\
\hline
\end{tabular}

Fuente: SPSS, elaboración del autor 
En este modelo se encontró que las variables ROE (Return on equity), riesgo y tangibilidad de los activos tuvieron una relación importante como determinantes de la variable dependiente, la estructura financiera. El ROE mantuvo una relación de carácter positivo, lo que mostraría que aquellas empresas que tuvieron mayores rendimientos sobre su patrimonio, tuvieron una más alta proporción de financiación con deuda que con patrimonio. El riesgo también tuvo una relación positiva con la proporción de deuda, lo que indicaría que las empresas con mayor volatilidad en su rentabilidad, presentaron mayor financiamiento a través de recursos externos que propios. $\mathrm{Y}$ en cuanto a la tangibilidad de los activos, se encontró una relación de carácter negativo, lo que significa que las empresas que mostraron mayores niveles de activos fijos, se financiaron en menor medida con recursos externos o pasivos.

\section{ANÁLISIS DE LOS RESULTADOS}

Los resultados obtenidos a partir de los tres modelos utilizados, permiten identificar algunas características de la estructura de capital de las medianas empresas establecidas en el departamento de Boyacá, además de los factores determinantes en la selección de sus fuentes de financiamiento.

En la Tabla 11 se presenta un comparativo de los tres modelos utilizados, las determinantes sobre las cuales se explica el comportamiento de la estructura, la naturaleza de sus relaciones y el coeficiente de cada una de ellas dentro de la ecuación del modelo.

Tabla 11. Variables con relación estadísticamente significativa y tendencia de la relación.

\begin{tabular}{|l|c|c|c|}
\hline VARIABLE & $\begin{array}{c}\text { MODELO 1 } \\
\text { Endeudamiento } \\
\text { total }\end{array}$ & $\begin{array}{c}\text { MODELO 2 } \\
\text { Endeudamiento } \\
\text { largo plazo }\end{array}$ & $\begin{array}{c}\text { MODELO 3 } \\
\text { Estructura } \\
\text { financiera }\end{array}$ \\
\hline Riesgo + (0.614) & $\mathrm{N} / \mathrm{S}$ & $+(0.609)$ & $-(0.525)$ \\
Tangibilidad de los activos & $-(0.740)$ & $\mathrm{N} / \mathrm{S}$ & $\mathrm{N} / \mathrm{S}$ \\
Crecimiento (Ventas) & $\mathrm{N} / \mathrm{S}$ & $+(0.785)$ & 0.803 \\
ROE N/S & $\mathrm{N} / \mathrm{S}$ & $+(0.578)$ & 0.768 \\
Coeficiente R cuadrado & 0.819 & 0.76 & 0 \\
\hline
\end{tabular}

Fuente: SPSS, elaboración del autor

Los modelos que tuvieron una mejor capacidad de explicación del fenómeno estudiado fueron los modelos 1 y 3 , que tomaron el endeudamiento total y la estructura financiera como variables independientes, respectivamente, puesto que estas variables no hacen diferencia en el plazo de los pasivos. Por el contrario, el modelo 2, aunque tuvo amplia bondad de ajuste, solamente demostró relación 
estadísticamente significativa para una variable y fue el que presentó menor coeficiente de determinación.

Los resultados del modelo 2 pueden explicarse a partir de los bajos niveles de endeudamiento de largo plazo que tienen estas empresas; también pueden originarse en el orden en las preferencias sobre fuentes de financiamiento, en donde el patrimonio ocupa el primer lugar con una proporción del $51 \%$ en promedio, el endeudamiento de corto plazo se sitúa en el segundo lugar, con una tasa del $43 \%$ en promedio y por último el endeudamiento de largo plazo, con un promedio del $6 \%$.

En los modelos 1 y 3 se encuentra una relación positiva del riesgo con la estructura de capital, teniendo en cuenta la forma en que se calculó el riesgo, a partir de la volatilidad de la rentabilidad durante los periodos analizados, su desviación estándar. Esto implica, por lo tanto, que las empresas con una menor estabilidad en su desempeño operativo y financiero, o que este hecho en general, implica una mayor utilización del financiamiento por medio de deuda, o que este se incrementa en periodos donde se presenta inestabilidad en el desempeño, es decir, seguiría una tendencia de aumento en el riesgo.

En estos mismos modelos ( 1 y 3 ), la variable tangibilidad de los activos muestra una relación negativa con la estructura de capital, lo que implica que donde se cuenta con un menor volumen de activos fijos, existen menores niveles de endeudamiento, o que el endeudamiento fue mayor en aquellos casos donde se dispuso de un menor volumen de activos no corrientes (fijos); además, que las empresas con mayores niveles de activos no corrientes se financian principalmente con recursos propios, y esto pese a contar con la posibilidad de ofrecer mayores garantías a las entidades financieras para obtener financiamiento de largo plazo.

En el modelo 2, donde se midió la estructura de capital para el endeudamiento de largo plazo, el principal hallazgo es la marcada relación positiva de la variable crecimiento en ventas con este tipo de endeudamiento; circunstancia que comprueba el efecto favorable que puede generar el endeudamiento a un mayor término en aquellas empresas que lo utilizan.

Como se mencionó anteriormente, la fuente menos utilizada para financiamiento ha sido la deuda de largo plazo, pues se encontró que solamente el $25 \%$ de las empresas del panel presentó un endeudamiento de largo plazo por lo menos igual o superior al 10 $\%$ de todas sus fuentes de recursos, y en el restante $75 \%$ de las empresas se cuenta con proporciones muy bajas o no se tiene endeudamiento de largo plazo.

El tipo de relación y su significancia muestran el importante efecto del apalancamiento como coadyuvante en el crecimiento de las empresas, además se constituye en una señal favorable acerca de la situación financiera de una empresa, puesto que refleja la confianza de los acreedores en su capacidad de pago, solidez y estabilidad en un horizonte más 
amplio de tiempo. Igualmente es un hecho que confirma las hipótesis acerca de las decisiones en cuanto a la estructura de capital, que tienen la capacidad de generar valor para la empresa, afirmación que fue hecha por Miller y Modigliani en su proposición 2 .

\section{CONCLUSIONES}

A partir del análisis de los diferentes modelos utilizados, se llega a las siguientes conclusiones:

Con respecto a las fuentes de financiamiento, las empresas prefirieron financiarse con recursos propios, en segundo lugar con endeudamiento de corto plazo y en tercer y último lugar, utilizaron el endeudamiento de largo plazo. Este tipo de recurso únicamente fue utilizado de forma representativa por el $25 \%$ de las empresas.

Las variables tangibilidad de los activos, riesgo, crecimiento en ventas y rentabilidad del patrimonio, fueron las únicas que mostraron una relación importante con la determinación de la estructura de capital, de acuerdo con los resultados de los diferentes modelos aplicados.

La tangibilidad de los activos mostró una relación negativa con el endeudamiento de corto plazo y la proporción deuda/recursos propios, que para el estudio se denominó estructura financiera, lo que mostró una baja utilización del endeudamiento cuando las empresas contaron con mayor volumen de propiedad, planta y equipo, pese a contar entonces con mejores garantías para respaldar sus deudas. En los estudios realizados en empresas de mayor tamaño o que cotizan en bolsa, la relación ha sido principalmente de carácter positivo, lo que muestra un comportamiento diferente en el financiamiento de las pymes.

El riesgo tuvo una relación positiva con el endeudamiento, lo que permite concluir que las empresas con mayores variaciones en su rentabilidad, recurrieron en mayor medida al endeudamiento como fuente de financiación. En escenarios de mayor riesgo, buscaron en mayor medida financiarse con deuda.

El crecimiento en ventas se relacionó positivamente con el endeudamiento de largo plazo, situación que evidenció las ventajas que otorga el endeudamiento como generador de valor, factor que favorece el crecimiento de las empresas que lo utilizan más adecuadamente.

En último término, se quiere resaltar la posibilidad de profundizar la investigación para desarrollar líneas de trabajo que permitan identificar y resolver los problemas de acceso a los recursos de deuda y de capital como fuentes adicionales de financiamiento. Este es un trabajo que además implica acciones gubernamentales, de los gremios económicos, del sector financiero y de los propios empresarios y emprendedores, de tal forma que se profundice el acceso a los mercados, se puedan eliminar las barreras de toda índole y se fomente la flexibilidad de las estructuras de financiamiento principalmente para este tipo de empresas. 


\section{REFERENCIAS}

1. Aybar-Arias, C., Casino-Martínez, A. \& López-Gracia, J. (2012). On the adjustment speed of SMEs to their optimal capital structure. Small Bus Econ, 2012(39), 977-996. doi: 10.1007/ s11187-011-9327-6

2. Baker, M. \& Wurgler, J. (2002). Market timing and capital structure. The Journal of Finance, 57(1), 1-32.

3. Barona, B. \& Gómez, A. (2010). Aspectos conceptuales y empíricos de la financiación de nuevas empresas en Colombia. Cuadernos de Administración, 43, 18.

4. Berumen, J., García, P. \& Domenge, R. (2012). Determinantes de la estructura de capital en la pequeña y mediana empresa familiar en México. Contaduría y Administración, 57(3), 67-96.

5. Boot, A. W. A. \& Thakor, A. V. (2011). Managerial autonomy, allocation of control rights, and optimal capital structure. The Review of Financial Studies, 24(10), 3434-3485.

6. Bradley, M., Jarrell, G. A. \& Kim, E. H. (1984). On the existence of an optimal capital structure theory and evidence. The Journal of Finance, 39(3), 857-879.

7. Comisión Económica para América Latina y el Caribe CEPAL. (2011). Política pública e instrumentos de financiamiento a las pymes en Colombia. Santiago de Chile: CEPAL.

8. Dang, V. A., Kim, M. \& Shin, Y. (2012). Asymmetric capital structure adjustments: New evidence from dynamic panel threshold models. Journal of Empirical Finance, 10, 405482.

9. Departamento Administrativo Nacional de Estadística DANE. (2012). Informe de coyuntura económica regional ICER Boyacá 2012. Bogotá: DANE - Banco de la República.

10. Forte, D., Barros, L. A. \& Nakamura, W. T. (2013). Determinants of the capital structure of small and medium sized Brazilian enterprises. Brazilian Administration Review, 10(3), 347-369.

11. Frank, M. \& Goyal, V. (2008). Trade-off and pecking order theories of debt. In B.E. Eckbo (ed.). Handbook of corporate finance: empirical corporate finance (pp. 1-85). Amsterdam: Elsevier/North-Holland.

12. Leary, M. (2009). Bank loan supply, lender choice, and corporate capital structure. The Journal of Finance, 64(3), 1143-1185. 
13. Lemmon, M., Roberts, M. \& Zender, J. (2008). Back to the beginning: persistence and the cross-section of corporate capital structure. The Journal of Finance, 63(4), 1575-1608.

14. Macas-Nunes, P., Serrasqueiro, Z., Nunes, A. \& Mendes, L. (2013). The relationship between growth of companies and labour productivity in Portuguese SMES: a dynamic panel data approach. Transformations in Business \& Economics, 12(3), 20-39.

15. Miller, M. H. \& Modigliani, F. (1958). The cost of capital, corporation finance and the theory of investment. The American Economic Review, 48(3), 261-297.

16. Miller, M. H. \& Modigliani, F. (1963). Corporate income taxes and the cost of capital: a correction. The American Economic Review, 53(3), 433-443.

17. Ministerio de Comercio, Industria y Turismo (2012). Perfil económico del departamento de Boyacá. Oficina de Estudios Económicos. Recuperado de: http://www.mincit.gov.co/ publicaciones.php?id=16724

18. Morellec, E., Nikolov, B. \& Schürhoff, N. (2012). Corporate governance and capital structure dynamics. The Journal of Finance, 67(3), 803-848.

19. Myers, S. C. (1984). The capital structure puzzle. The Journal of Finance, 39(3), 575-592.

20. Myers, S. C. \& Majluf, N. S. (1984). Corporate financing and investment decisions when firms have information that investors do not have. Journal of Financial Economics, 13, $187-221$.

21. Palacín-Sánchez, M. J., Ramírez Herrera, L. M. \& Di Pietro, F. (2013). Capital structure of SMEs in Spanish Regions. Small Bus Econ, 2013(41), 503-519. doi: 10.1007/s11187012-9439-7

22. Psillaki, M. \& Daskalakis, N. (2009). Are the determinants of capital structure country or firm specific? Small Bus Econ, (33), 319-333. doi: 10.1007/s11187-008-9103-4

23. Qian, Y., Tian, Y. \& Wirjanto, T. (2009). Do Chinese publicly listed companies adjust their capital structure toward a target level? China Economic Review, 20, 662-676.

24. Stephanou, C. \& Rodríguez, C. (2008). Colombia financiamiento bancario para las pequeñas y medianas empresas (pyme). En U. d. g. p. M. y. C. O. R. p. L. y. e. Caribe (Ed.). Bogotá: Banco Mundial.

25. Shyam-Sunder, L. \& Myers, S. (1998). Testing static tradeoff against pecking order models of capital structure. Journal of Financial Economics, 51, 219-244.

26. The World Bank \& Internacional Finance Corporation. (2011). Colombia Country Profile 2010. Washington: World Bank. 
La estructura de capital en las medianas empresas del departamento de Boyacá Andrés Fernando Mejía Amaya

ANEXO 1

MUESTRA DE EMPRESAS

\begin{tabular}{|c|l|}
\hline NIT & \multicolumn{1}{|c|}{ NOMBRE O RAZÓN SOCIAL } \\
\hline 800240258 & ALBORAUTOS LTDA. \\
826002217 & ALMACENES SERGO LTDA. \\
800022005 & AUTOBUSES AGA DE COLOMBIA LTDA. \\
826000361 & AUTOSERVICIO PARAÍSO S.A. \\
830092048 & CASAGRO S.A. \\
800092661 & COELCI LTDA. \\
800209179 & COLOMBIANA DE ENCOMIENDAS S.A. \\
891855600 & DISTRIBUIDORA DE COMBUSTIBLES EL SOL LTDA. \\
891857733 & DISTRIBUIDORA TROPIBOY LTDA. \\
891855573 & EMPRESA DE FOSFATOS DE BOYACÁ S.A. \\
891801951 & FAMA S.A. \\
832000123 & FERAUTOS LTDA. \\
820004492 & INDUSTRIA DE LICORES DE BOYACÁ S.A. -C.I. \\
860053330 & INMUNIZADORA DE MADERAS DEL ORIENTE LTDA. \\
891855774 & INVERSIONES BOYACÁ LTDA. \\
820000187 & INVERSIONES LA PRADERA LTDA. \\
891801193 & INVERSIONES LADRILLOS MAGUNCIA S.A. \\
891855859 & INVERSIONES Y PROMOCIONES LTDA. \\
800116325 & JAIME PARRA P. Y CIA. LTDA. \\
891800215 & NOGO BOYACÁ LTDA. \\
891856506 & PRODUCTORA DE ALAMBRES S.A. \\
800188412 & SANOHA LTDA. MINERÍA MEDIO AMBIENTE Y FORESTAL \\
820000045 & SERVIAGROFINCA LTDA. \\
891857724 & UNIÓN NACIONAL DE DROGUERÍAS LTDA. \\
\hline
\end{tabular}

